\title{
Axonal Ensheathment and Septate Junction Formation in the Peripheral Nervous System of Drosophila
}

\author{
Swati Banerjee, ${ }^{1}$ Anilkumar M. Pillai, ${ }^{1}$ Raehum Paik, ${ }^{1}$ Jingjun Li, ${ }^{2}$ and Manzoor A. Bhat ${ }^{1,2,3,4}$ \\ ${ }^{1}$ Department of Cell and Molecular Physiology, ${ }^{2}$ Curriculum in Neurobiology, ${ }^{3}$ University of North Carolina Neuroscience Center, and \\ ${ }^{4}$ Neurodevelopmental Disorders Research Center, University of North Carolina School of Medicine, Chapel Hill, North Carolina 27599-7545
}

\begin{abstract}
Axonal insulation is critical for efficient action potential propagation and normal functioning of the nervous system. In Drosophila, the underlying basis of nerve ensheathment is the axonal insulation by glial cells and the establishment of septate junctions (SJs) between glial cell membranes. However, the details of the cellular and molecular mechanisms underlying axonal insulation and SJ formation are still obscure. Here, we report the characterization of axonal insulation in the Drosophila peripheral nervous system (PNS). Targeted expression of tau-green fluorescent protein in the glial cells and ultrastructural analysis of the peripheral nerves allowed us to visualize the glial ensheathment of axons. We show that individual or a group of axons are ensheathed by inner glial processes, which in turn are ensheathed by the outer perineurial glial cells. SJs are formed between the inner and outer glial membranes. We also show that Neurexin IV, Contactin, and Neuroglian are coexpressed in the peripheral glial membranes and that these proteins exist as a complex in the Drosophila nervous system. Mutations in neurexin IV, contactin, and neuroglian result in the disruption of blood-nerve barrier function in the PNS, and ultrastructural analyses of the mutant embryonic peripheral nerves show loss of glial SJs. Interestingly, the murine homologs of Neurexin IV, Contactin, and Neuroglian are expressed at the paranodal SJs and play a key role in axon-glial interactions of myelinated axons. Together, our data suggest that the molecular machinery underlying axonal insulation and axon-glial interactions may be conserved across species.
\end{abstract}

Key words: peripheral glia; glial septate junctions; chordotonal organs; blood-nerve barrier; neuron-glial interactions; cell adhesion

\section{Introduction}

Glial ensheathment of axons ensures proper action potential propagation. During evolution, species developed mechanisms to enhance the conduction velocity either by increasing the axon diameter (for example, the squid giant axon) or by producing a multilayered myelin membrane sheath around the axon as seen in the vertebrates (Hodes, 1953; Peters et al., 1991). Myelination provided additional advantages in establishing specific axon-glial interactions to organize axons into unique molecular domains to promote saltatory conduction (Bhat, 2003; Salzer, 2003).

In the fruit fly, Drosophila melanogaster, many types of glial cells have been identified based on their anatomical features and putative functions (Ito et al., 1995; Freeman et al., 2003). In the absence of a myelin sheath and nodes of Ranvier, Drosophila axons are wrapped by peripheral (inner) glial cells, which in turn are wrapped by much larger perineurial (outer) glial cells (Bellen et al., 1998). The inner glial cell processes wrap the axons, which

Received Dec. 16, 2005; revised Feb. 13, 2006; accepted Feb. 15, 2006.

This work was supported by National Institute of General Medical Sciences Grant GM63074 and National Institutes of Health-National Cancer Institute Grant CA78437 (M.A.B.) and by funds from the State of North Carolina. We thank E. Raff (Indiana University) for reagents; V. Madden (University of North Carolina EM Facility) for technical assistance; A. Sousa for the schematic in Figure 5 and supplemental data in Figure 2S; and V. Budnik, A. Fanning, and members of the Bhat laboratory for comments and discussions.

Correspondence should be addressed to Dr. Manzoor A. Bhat, Department of Cell and Molecular Physiology, Neuroscience Research Building 5109F, University of North Carolina School of Medicine, Chapel Hill, NC 27599-7545. E-mail:manzoor_bhat@med.unc.edu.

DOI:10.1523/JNEUROSCI.5383-05.2006

Copyright $\odot 2006$ Society for Neuroscience $\quad$ 0270-6474/06/263319-11\$15.00/0 in turn are ensheathed by the outer glial membrane. This insulation protects the neural microenvironment from the high potassium levels of the hemolymph (Hoyle, 1952). In addition to axonal ensheathment, the glial membranes are also involved in the formation of cellular junctional complexes at the membrane interfaces between either two glial cells or between axons and glial cells. One such junctional complex displays a unique ladder-like structure characteristic of the septate junctions (SJs). In invertebrate species, SJs provide the functional equivalent of vertebrate tight junctions (TJs) to maintain the blood-brain barrier (BBB) (Bellen et al., 1998; Carlson et al., 2000; Tepass et al., 2001). Loss or disruption of SJs resulting from mutations in genes encoding SJ proteins leads to the breakdown of the barrier function, ultimately resulting in paralysis (Auld et al., 1995; Baumgartner et al., 1996). Recent analyses of SJs in Drosophila show the presence of a number of proteins that are enriched at or associated with SJs (for review, see Banerjee et al., 2006). Interestingly, most of these proteins are localized at the epithelial SJs, only Neurexin IV (Nrx IV) and Gliotactin are reported to be functional in the peripheral glia, whereas Moody and Loco are required in the surface glia to achieve effective insulation of the CNS (Bainton et al., 2005; Schwabe et al., 2005).

Although axo-glial SJs and their molecular components have been identified in vertebrates, the molecular components of Drosophila SJs in the nervous system and their functions are still not fully understood. Here, we report on the cellular basis of axonal ensheathment in the Drosophila peripheral nerves and show that 
the inner glial membrane processes wrap the axons. We show that SJs are established between the outer and inner glial membranes and that these junctions are continuously present along the length of the nerve fibers. Ultrastructural and dye exclusion analyses of $n r x I V$, contactin (cont), and neuroglian ( $n r g$ ) mutants revealed loss of SJs in the peripheral nerves and a breakdown of the blood-nerve barrier (BNB) in the PNS, respectively. Our studies provide evidence for an evolutionarily conserved molecular complex at the SIs and provide a genetic basis to study axonal insulation and axon-glial interactions in a Drosophila model system.

\section{Materials and Methods}

Drosophila stocks. $n r x I V^{4304}$, cont ${ }^{e x 956}$, and $n r g^{1}$ alleles have been described previously (Baumgartner et al., 1996; Faivre-Sarrailh et al., 2004). Upstream activating sequences (UAS)-tau green fluorescent protein (GFP) was obtained from S. Crews (University of North Carolina, Chapel Hill, NC), repo-GAL4 was obtained from V. Auld (University of British Columbia, Vancouver, Canada), and $\mathrm{gcm}^{\triangle P 1}$ was obtained from B. Jones (University of Mississippi, Oxford, MI). All other reagents used in the current study were obtained from the Drosophila Stock Center (Bloomington, IN).

Generation of antibodies and immunofluorescence analysis. For the production of polyclonal antibodies against Reverse polarity (Repo) and Nrg, cDNA fragments encoding C-terminal 295 and 140 amino acids, respectively, were cloned in a his-tag vector $p E T 28 a(+)$ (Novagen, Madison, WI) and expressed in Escherichia coli BL21DE3. The recombinant proteins were purified on cobalt columns and used for the immunization of guinea pigs and rats as described previously (Bhat et al., 1996).

Antibody staining of embryos was performed as described previously (Bhat et al., 1999). Primary antibodies used were rabbit anti-Nrx IV (Baumgartner et al., 1996), guinea pig anti-Cont (Faivre-Sarrailh et al., 2004), rabbit anti-GFP (Invitrogen, San Diego, CA), mouse anti-FAS II (Developmental Studies Hybridoma Bank, University of Iowa, Iowa City, IA), rabbit anti- $\beta 3$ tubulin (Dettman et al., 2001), rat anti-Nrg (our study), and guinea pig anti-Repo (our study). All secondary antibodies used in this study were from Jackson ImmunoResearch (West Grove, PA). Images were captured on Bio-Rad (Hercules, CA) Radiance 2000 confocal microscopes and processed with Adobe PhotoShop software (Adobe Systems, San Jose, CA).

Transmission electron microscopy and data analysis. For transmission electron microscopy (TEM), both wild-type and mutant embryos from various genotypes were dechorionated. Homozygous mutant embryos were screened against GFP from green balancers and processed for TEM according to published procedures (Auld et al., 1995). Wandering thirdinstar wild-type larvae were also fixed and processed according to Auld et al. (1995). Analyses of embryonic wild-type nerves were based on three wild-type (Canton S) samples and three each for mutants of nrx IV and $n r g$. All of the samples were sectioned in a transverse plane. Overall, $\sim 250$ images were photographed and analyzed for each of the samples. Mutant samples were processed for TEM in parallel with wild-type samples in three separate batches to minimize variations resulting from processing and fixation conditions. Serial sections were performed on the abdominal nerves of the wandering third-instar larvae. Approximately $50 \mathrm{semi}-$ thin $(70 \mathrm{~nm})$ serial sections of the larval peripheral nerve fibers were analyzed for the presence of SJs within $\sim 3.5 \mu \mathrm{m}$ region of the nerve.

Biochemical methods and immunoprecipitation. Wild-type fly heads were homogenized in $10 \mathrm{ml}$ of ice-cold buffer containing $50 \mathrm{~mm}$ Tris$\mathrm{HCl}, \mathrm{pH}$ 7.6, $3 \mathrm{~mm} \mathrm{MgCl}_{2}, 320 \mathrm{~mm}$ sucrose, and protease inhibitors using a glass homogenizer. The homogenates were centrifuged at $1000 \times g$ for $15 \mathrm{~min}$ at $4^{\circ} \mathrm{C}$. The supernatants were centrifuged again at 100,000 $\times \mathrm{g}$ for $2 \mathrm{~h}$ at $4^{\circ} \mathrm{C}$. The membrane pellets were extracted for $30 \mathrm{~min}$ on ice in buffer containing $50 \mathrm{~mm}$ Tris- $\mathrm{HCl}, \mathrm{pH}$ 7.6, 2\% sucrose monolaurate, $0.1 \%$ sodium deoxycholate, and protease inhibitors. The solubilized pellets were centrifuged again at $100,000 \times g$ for $2 \mathrm{~h}$ at $4^{\circ} \mathrm{C}$. The supernatants were used for the immunoprecipitation. The Protein A beads $(100 \mu \mathrm{l})$ were preincubated with $8 \mu \mathrm{l}$ of either Nrx IV, Cont, or Nrg antibodies for $3 \mathrm{~h}$ and washed three times with PBS. The antibody-Protein A beads were added to $1.5 \mathrm{ml}$ of the solubilized membrane fraction and incubated overnight at $4^{\circ} \mathrm{C}$. The beads were washed four times with $50 \mathrm{~mm}$ Tris$\mathrm{HCl}$, pH 7.6, for $10 \mathrm{~min}$ each. The protein complexes bound to the beads were eluted in $150 \mu \mathrm{l}$ of $100 \mathrm{~mm}$ glycine- $\mathrm{HCl}, \mathrm{pH} 2.5$. Twenty microliters of the samples from each genotype were separated on SDS-PAGE for immunoblotting with respective antibodies.

Sucrose density gradient centrifugation. Fly heads were homogenized in $10 \mathrm{~mm}$ Tris-HCl, pH 7.4, containing $100 \mathrm{~mm} \mathrm{NaCl}, 10$ mм EDTA, $1 \mathrm{~mm}$ PMSF, and $0.5 \%$ Triton X-100 with protease inhibitors followed by centrifugation at $100,000 \times g$ for $20 \mathrm{~min}$ at $4^{\circ} \mathrm{C}$. After protein estimation, 2 $\mathrm{mg}$ of total protein was layered on a $10-45 \%$ continuous sucrose gradient followed by centrifugation at $100,000 \times g$ for $20 \mathrm{~h}$ at $4^{\circ} \mathrm{C}$. Fractions of $250 \mu \mathrm{l}$ were collected from the top, and appropriate amounts were processed for immunoblotting as described above.

Dye exclusion assay. Stage 16-17 embryos were collected and prepared for dye injections as described previously (Lamb et al., 1998). Rhodamine (30 mg/ml)-labeled dextran (10,000 molecular weight, neutral; Invitrogen) in injection buffer ( $5 \mathrm{~mm} \mathrm{KCl,} 0.1 \mathrm{~mm}$ sodium phosphate, $\mathrm{pH} 6.8$ ) was injected into the body cavity at the posterior end of the embryo by using an Eppendorf (Hamburg, Germany) micromanipulator FemtoJet. The embryos were visualized directly under a confocal microscope, and images were captured simultaneously within a specified time. For wildtype and for each mutant (counter selected against a GFP balancer), 10 embryos and 18 embryos, respectively, were injected with the rhodamine-labeled dextran dye.

Statistics. Statistical analysis was performed using Student's $t$ tests. Data are expressed as the means \pm SEM.

\section{Results}

Axonal ensheathment of the Drosophila embryonic and larval peripheral nerves

In the Drosophila embryonic nervous system, $\sim 10 \%$ of the cells constitute glial cells of various types and function. They have been classified using various enhancer trap markers that identify specific subsets of glial cells (Ito et al., 1995). To understand the anatomical and cellular basis of axonal insulation, knowledge of the spatiotemporal distribution of the ensheathing glial cells and their membrane processes along the axons in the nervous system is essential. We analyzed the general distribution of glial cells at three key developmental stages [stages according to CamposOrtega and Hartenstein (1997)] to determine the relationship and pattern of peripheral glial cells and the nerve fibers. To visualize glial cell distribution at embryonic developmental stages 13, 14 , and 16, we used antibodies against a glial-specific homeodomain protein, Repo, which is expressed exclusively in glial cell nuclei (Fig. 1A,D,G, green) (Campbell et al., 1994) and a cell adhesion molecule, Fasciclin II, which is expressed in the motor axons (Fig. 1A-I, red). At stage 13, most of the glial cells have been specified. The peripheral glial cells are still present at the nerve exit at the CNS-PNS border (Fig. $1 A-C$; white lines in $C, F$, and $I$ represent the CNS-PNS border). To determine the extent of the glial cytoplasm and glial processes at stage 13, we used repo:GAL4::UAS:tau-GFP combination to express tau-GFP in the glial cells to aid in the visualization of the glial cytoplasm and glial processes, because tau-GFP binds to the microtubular network (Williams et al., 2000). As shown in Figure $1 B$ and at a higher magnification in Figure $1 C$, immunostaining against GFP revealed that glial cytoplasm is already surrounding the nerve fibers and that peripheral glia are positioned into a cone shape ready to move toward the periphery (Fig. $1 C$, arrow). At stage 14, the peripheral glial cells have completed their proliferation and 8-10 glial cells per abdominal hemisegment lineup along the nerve tracts (Jones et al., 1995; Sepp et al., 2000). The peripheral glial cell nuclei are present on the nerve tracts and are better separated from each other compared with stage 13 (Fig. 1, compare $A$ and $D)$. Immunostaining of repo:GAL4::UAS:tau-GFP 

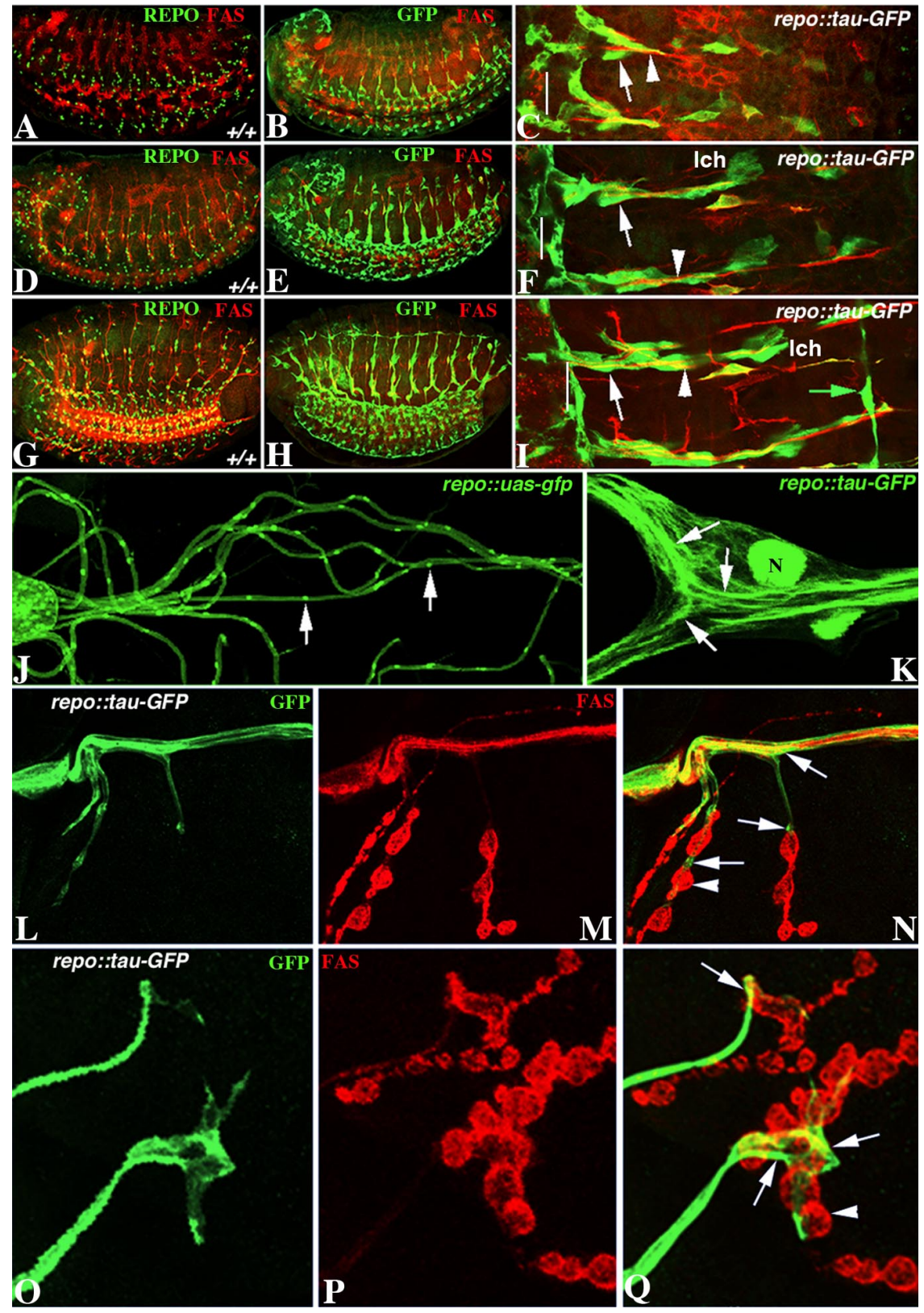

Figure 1. Glial cell profile in the embryonic and larval PNS. $\boldsymbol{A}-\boldsymbol{C}$, Stage 13 wild-type $(\boldsymbol{A})$ and repo-GAL4::UAS-tauGFP ( $\boldsymbol{B}, \boldsymbol{C})$ embryos stained with anti-Repo (green; $\boldsymbol{A}$ ) and anti-Fas II (red; $\boldsymbol{A}-\boldsymbol{C}$ ) show that the clusters of peripheral glial cells begin to migrate to the periphery along the motor axon tracts. The anti-GFP (green; $\boldsymbol{B}, \boldsymbol{C}$ ) shows the profile of the glial microtubular cytoskeleton, thus highlighting the glial process extension. $\boldsymbol{C}$, Higher magnification of a portion of the embryo shown in $\boldsymbol{B}$ reveals that the glial cytoplasmic processes (arrow) extend along the motor axon tracts (arrowhead) to reach the periphery. The CNS -PNS boundary is highlighted by a white line $(\boldsymbol{C}, \boldsymbol{F}, \boldsymbol{I})$. D-F, Stage 14 wild-type $(\boldsymbol{D})$ and repo-GAL4::UAS-tauGFP $(\boldsymbol{E}, \boldsymbol{F})$ embryos show Repo-positive glial cells (green; $\boldsymbol{D}$ ) moving further into the periphery along the motor axons (red; $\boldsymbol{D})$. The tau-GFP visualized by anti-GFP staining (green; $\boldsymbol{E}, \boldsymbol{F}$ ) shows the extent of the glial microtubular network (arrow; $\boldsymbol{F}$ ) along the axonal tracts (arrowhead; $\boldsymbol{F}$ ). The group of lateral chordotonal sensory organs (Ich) is also visible in this focal plane and shows expression of GFP in ligament cells, which ensheath chordotonal neurons. $\mathbf{G}-\mathbf{I}$, Stage 16 wild-type (G) and repo-GAL4::UAS-tauGFP $(\boldsymbol{H}, \boldsymbol{I})$ embryos show that the Repo-positive glial cells (green; $\boldsymbol{G}$ ) have migrated to the periphery, and the glial processes ( $(g r e e n ; \boldsymbol{H}, \boldsymbol{I}$ ) visualized by anti-GFP staining show near complete ensheathment of the peripheral nerve (arrow; $\boldsymbol{I}$ ) axon tracts (red; $\boldsymbol{H}, \boldsymbol{I})$. The glial ensheathment has extended up to Ich. The axon tracts are seen running through the glial cells. The sensory bipolar dendritic neuron is also ensheathed by its supporting glial cell (green arrow). J, A portion of the third-instar larval ventral nerve cord with peripheral nerves expressing GFP under repo-GAL4 driver. The glial nuclei (arrows) are randomly lined up along the peripheral nerves, and no consistent distance is observed between neighboring glial cells. $\boldsymbol{K}$, A portion of an abdominal nerve with a glial cell nucleus (N) from repo-GAL4::UAS-tauGFP stained with anti-GFP reveals the pattern of the glial microtubular network (arrows) with tracts of microtubules separated from each other. $\boldsymbol{L}-\boldsymbol{Q}$, A portion of a peripheral nerve of repo-GAL4::UAS-tauGFP stained with anti-GFP $(\boldsymbol{L}$, $\mathbf{O})$ and anti-Fas II $(\boldsymbol{M}, \boldsymbol{P})$ reveals the ensheathment of the nerve by the glial membranes $(\boldsymbol{N}, \mathbf{Q})$. The glial membrane extends partly into the initial portion of the third-instar larval neuromuscular junctions (arrows; $N, Q$ ) (Sepp et al., 2000). The glial processes also display arborizations at the initial contact site with the muscle ( $\mathbf{Q}$, arrows). The Fas II marked synaptic boutons (arrowheads; $\boldsymbol{N}, \mathbf{Q}$ ) that are away from the nerve entry site are devoid of glial insulation and are protected by the musculature. stage 14 embryos shows that glial cells are further along the axon tracts and are surrounding the axons (Fig. $1 E$ ). At a higher magnification, the glial cell bodies and processes (Fig. $1 F$, green, arrow) are seen ensheathing the axon tracts (red, arrowheads). At stage 16, the migration of the peripheral glial cells (green) along the nerve tracts (red) is complete (Fig. 1G). Immunostaining of repo:GAL4::UAS:tau-GFP stage 16 embryos shows that glial cell bodies and processes have completely ensheathed the major axon tracts (Fig. $1 \mathrm{H}$ ). As shown in Figure $1 I$, at a higher magnification, the glial cell bodies and processes (green, arrows) are seen ensheathing the axons tracts (red, arrowheads). The ensheathment of lateral chordotonal sensory neurons (lch) by the ligament cells is also highlighted by tau-GFP. Similarly, a bipolar dendritic neuron is also ensheathed by its cognate glial cell (green arrow). These data show that glial ensheathment of the axons begins as soon as the peripheral glial cells exit the CNS, and in the later stages of development, the glial cells continue to migrate along the axon tracts and stretch their processes for long distances to insulate the embryonic peripheral nerves.

We next analyzed the glial distribution along the third-instar larval abdominal nerves to determine how larval nerves are insulated given that some of these nerves run almost the entire length of the larval body. The larval nerves exit the ventral nerve cord and innervate the musculature of each individual abdominal segment (Leiserson et al., 2000). As shown in Figure 1J, GFP fluorescence of the repo:GAL4::UAS:GFP third-instar larvae allowed us to visualize the glial cells on the nerve tracts. Most of the GFP protein remains in the cell body, but some of the GFP is transported along the glial membranes, which highlight the nerve tracts. The glial cells are present along the length of the peripheral nerves and do not display any uniform distribution along the nerves (Fig. $1 \mathrm{~J}$, arrows). To determine the extent of glial cytoplasm and glial microtubules, we immunostained repo:GAL4::UAS:tau-GFP third-instar larval abdominal nerves (Fig. $1 K$ ). This revealed an extensive microtubular network in the glial cytoplasm (Fig. $1 \mathrm{~K}$, arrows; $\mathrm{N}$ is a glial nucleus), indicating that glial cytoplasm is distributed to accommodate axonal fascicles during ensheathment. To further analyze axonal ensheathment of the abdominal nerves at the level of the neuromuscular attachment sites between glial cells, axons, and the musculature, we immunostained repo:GAL4::UAS:tau-GFP third-instar abdominal nerves with anti-GFP (Fig. $1 L, O$, green) and anti-Fas II to highlight axons and synapses (Fig. $1 M, P$, red). This re- 
vealed that the peripheral nerve axons are ensheathed by the glial membrane processes and that glial ensheathment in larval nerves extends partly to neuromuscular synapses (Fig. $1 N, Q$, merge) (Sepp et al., 2000). In addition, glial processes form arborizations at the initial areas of the neuromuscular synapses (Fig. 1Q, arrows; arrowheads point to synaptic boutons in $N, Q)$. Together, the immunofluorescence data show that axonal ensheathment in the Drosophila PNS is performed by ensheathing peripheral glial cells.

\section{Ultrastructural analysis of the axonal ensheathment and the presence of septate junctions in the peripheral nerves}

The light microscopic analysis of the peripheral glial distribution on the embryonic and larval abdominal nerves provided a gross anatomical perspective. We wanted to further analyze the ultrastructural features of the embryonic and larval peripheral nerves with respect to the nature of axonal ensheathment, the arrangement of axon fascicles and the presence of cellular junctions. As shown in Figure $2 A$, a cross section of an embryonic peripheral nerve at low magnification revealed many single axons (Fig. 2, a) or a group of axons surrounded by glial membrane processes of the inner glial cell (Fig. 2, g). Such an arrangement of axon fascicles has been described previously (Juang and Carlson, 1994; Auld et al., 1995). After closer examination, the glial membranes (inner and outer) come in close apposition to establish electron-dense junctional structures, which represent the glial-glial SJs (Fig. $2 \mathrm{~A}$, arrow). Next, we analyzed the ultrastructure of a third-instar larval abdominal nerve. As shown in Figure $2 B$, the cross section of the larval nerve revealed the presence of $\sim 80-90$ axons (a) tightly held together and ensheathed by inner glial membranes $(\mathrm{m})$. The outer or perineurial glial membrane (P) wraps around the nerve fiber, and areas where the outer and inner glial membranes come in close proximity show the presence of SJs (Fig. $2 B$, arrow). The nerve is surrounded by a neural lamella (Fig. 2, 1), which is secreted by the outer glial cells (Leiserson et al., 2000). Within the abdominal nerve, two types of insulations are observed: ensheathment of individual axons by the inner glial membrane and ensheathment of axon fascicles (a group of axons $=$ a fascicle) by the inner glial membrane. As shown in Figure $2 C$, axons (a) are surrounded by the membrane $(\mathrm{m})$ processes of the inner glial cells, thereby insulating individual axons. An entire fascicle that is ensheathed by the inner glial membrane $(\mathrm{m})$ indicated by arrowheads is shown in Figure $2 D$. All of these axons are held in register by the inner glial membrane (Fig. 2D, arrowheads). This pattern was consistently observed in 50 consecutive serial sections of third-instar
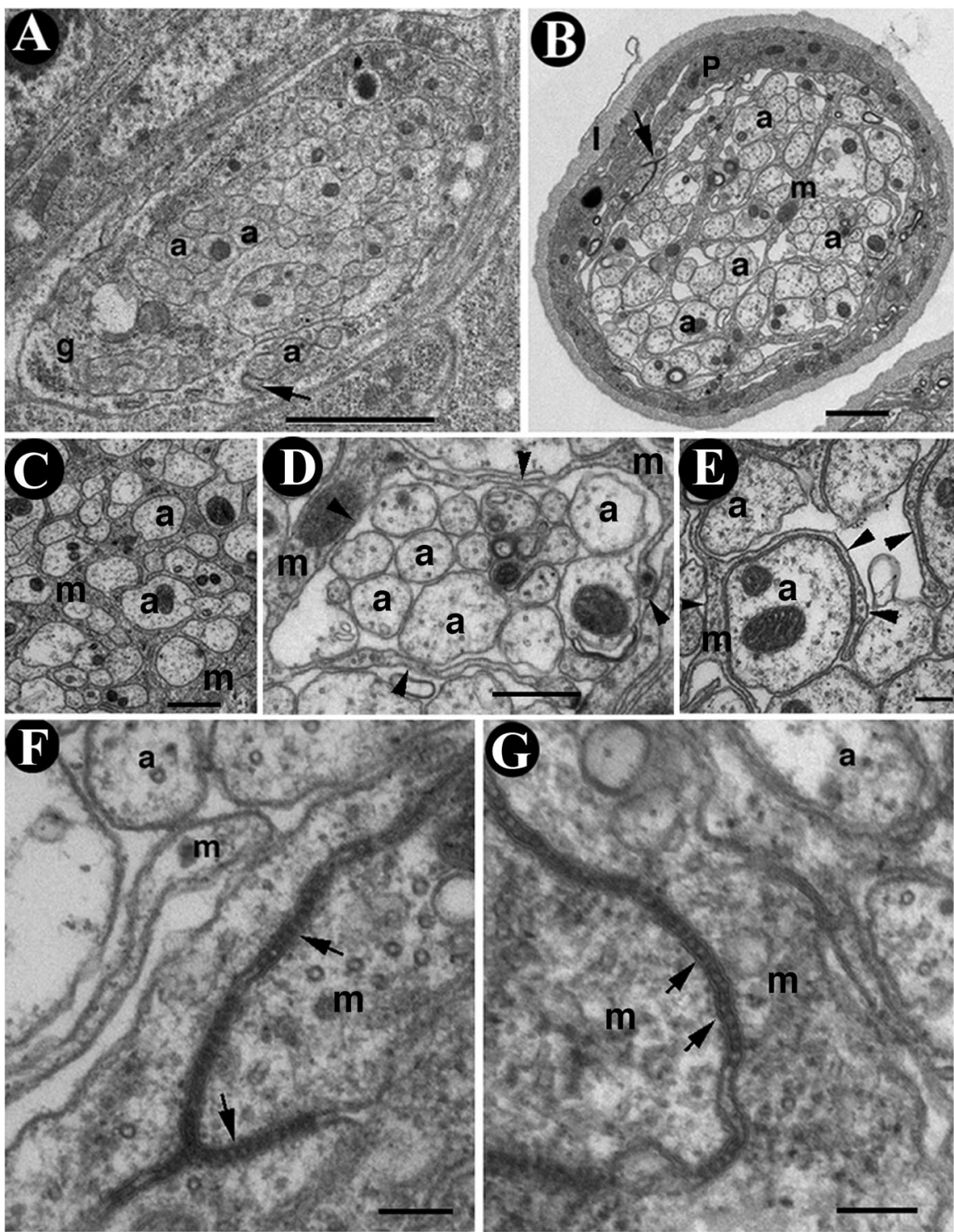

Figure 2. Axonal ensheathment and presence of junctions in the nerve fibers. A, Cross section through a 20- to 22-h-old wild-type embryonic nerve fiber showing tightly held axons (a) surrounded by a glial cell ( $\mathrm{g}$ ). The electron-dense region where two glial membranes come in close apposition is highlighted by an arrow. $\boldsymbol{B}$, Cross section through a wild-type third-instar peripheral nerve at a low magnification shows the arrangement of axons (a) surrounded by glial membranes ( $m$ ). The outer perineurial glial cell $(P)$ is surrounding the axons $(a)$ and the inner glial membranes $(m)$. The nerve is surrounded by a neural lamella (I). Note the presence of electron-dense areas that contain SJs (arrow). C, Section through a peripheral nerve showing glial membrane $(\mathrm{m})$ interspersed between the axons (a). $\boldsymbol{D}$, A nerve fascicle containing a group of axons is collectively wrapped by the inner glial membrane $(\mathrm{m})$. The arrowheads point to the boundary of the glial membrane that ensheaths this fascicle. Some axons (a) in the peripheral nerves are individually wrapped by the inner glial membrane ( $\boldsymbol{E}$, arrowheads). $\boldsymbol{F}$, A portion of the same nerve as in $\boldsymbol{B}$ at a higher magnification shows axons (a) surrounded by glial membrane processes ( $\mathrm{m}$ ). The inner and outer glial membranes come in close proximity to form the SJs (arrows). Glial membranes with SJs always display a distinct electron dense ladder-like structure corresponding to glial-glial SJs ( $\boldsymbol{F}, \boldsymbol{G}$, arrows). Scale bars: $A, D, 0.5 \mu \mathrm{m} ; \boldsymbol{B}, \boldsymbol{C}, 1 \mu \mathrm{m} ; \boldsymbol{E}, 0.25 \mu \mathrm{m} ; \boldsymbol{F}, \boldsymbol{G}, 0.1 \mu \mathrm{m}$.

peripheral nerve fiber (data not shown). An example of a single axon (a) that is ensheathed by the inner glial membrane is shown in Figure $2 E$. The arrowheads point to the membrane process that provides ensheathment to a single axon. These data indicate that Drosophila peripheral axons may be ensheathed individually like the Schwann cell myelinated peripheral axons of the vertebrates or collectively like the unmyelinated peripheral nerves of the vertebrates. Thus, ensheathment of axons does occur in the Drosophila peripheral nerves except that the membranes do not undergo multiple wraps as seen in vertebrate myelinated axons.

Next, we analyzed the areas of the peripheral nerves, where the 
inner and outer glial membranes come in close apposition to establish electron-dense cellular contacts. At a higher magnification, the region indicated by an arrow in Figure $2 B$ revealed distinct electron dense ladder-like structures between the outer and inner glial membranes $(\mathrm{m})$. These structures represent the glial-glial SJs characteristic of the pleated SJs (Fig. 2F, arrows) (Banerjee et al., 2006). A similar region from another abdominal nerve also shows the presence of ladder-like SJs between the outer and inner glial membranes $(\mathrm{m})$ with arrows pointing to individual septa of the ladder (Fig. 2G). The presence of SJs is consistently seen in the serial sections at the same region running along the length of the nerve fibers (supplemental Fig. $1 S$, available at www.jneurosci.org as supplemental material). Together, the ultrastructural analysis of the peripheral nerve fibers provided a detailed anatomical view of the axonal ensheathment by the glial cells and also revealed the presence of septate junctions between the glial membranes.

\section{Septate junction proteins Nrx IV, Cont, and Nrg are expressed in the Drosophila peripheral nerves}

The ultrastructural analysis of the peripheral nerves showed the presence of SJs. We established whether NRX IV, CONT, and NRG, which we have shown previously to localize to epithelial SJs (Faivre-Sarrailh et al., 2004), are expressed in the peripheral nerves and whether these proteins are expressed by axons or glia or both. To visualize the peripheral nerves of wild-type stage 16 embryos, we performed triple immunostaining using antibodies against Nrx IV (Fig. 3A, green), FasII (Fig. 3A-E, red), and Repo (Fig. $3 A-F$, blue). Nrx IV localization in the peripheral nerve is in the glial membrane (Fig. 3A, arrowhead, green) along FasIIlabeled motor axons (Fig. $3 A$, red, arrow), and Repo is localized to the glial nuclei (Fig. $3 A$, blue). Similarly, immunostaining against Cont showed that Cont is also expressed in the glial cells and localizes to the glial membrane (Fig. $3 B$, green, arrowhead) and shows no detectable expression in the axons (Fig. 3B, red, FasII arrow). In contrast, immunostaining against Nrg showed that Nrg is expressed in both the glial cells and the axons. The glial expression of $\mathrm{Nrg}$ is seen along the membranes (Fig. 3C, arrowheads), and coexpression with FasII is seen as yellow in the axons (Fig. 3C, arrow). Thus, the immunofluorescence analysis showed that the septate junction proteins Nrx IV, Cont, and Nrg are expressed in the peripheral nerves.

To further confirm the glial membrane localization of Nrx IV, Cont, and Nrg, we studied the localization of these three proteins in glial cells missing $(\mathrm{gcm}$ ) mutant embryos. $\mathrm{gcm}$ mutants retain very few glial cells in the embryos compared with wild type (Hosoya et al., 1995; Jones et al., 1995). gcm mutant embryos, identified by the loss of Repo-positive glial nuclear staining (Fig. $3 D-F$, blue), shows absence of glial membrane staining of Nrx (Fig. 3D, green), Cont (Fig. 3E, green), and $\mathrm{Nrg}$ (Fig. 3F, green). Long stretches of peripheral axons stained with either anti-Fas II (Fig. $3 D, E$, red) or anti-HRP (Fig. $3 F$, red) do not show any glial membrane ensheathment. However, Nrx IV, Cont, and Nrg show normal localization in the epithelial cells and trachea (Fig. 3, compare $D$ and $E$ with $A-C$, respectively). The axonal localization of $\mathrm{Nrg}$ is unaffected in $\mathrm{gcm}$ mutants (Fig. $3 F$ ). Together, these data demonstrate that Nrx IV, Cont, and Nrg are components of the glial membrane in the peripheral nerves, and Nrg has additional expression in the axons of the peripheral nerves.

Next, we performed the TEM analysis of the $\mathrm{gcm}$ mutant nerves to establish that the ensheathment of the axons is dependent on the peripheral glial cells. As shown in Figure $3 G$, in the wild-type nerves, axons (a) are properly ensheathed and the inner
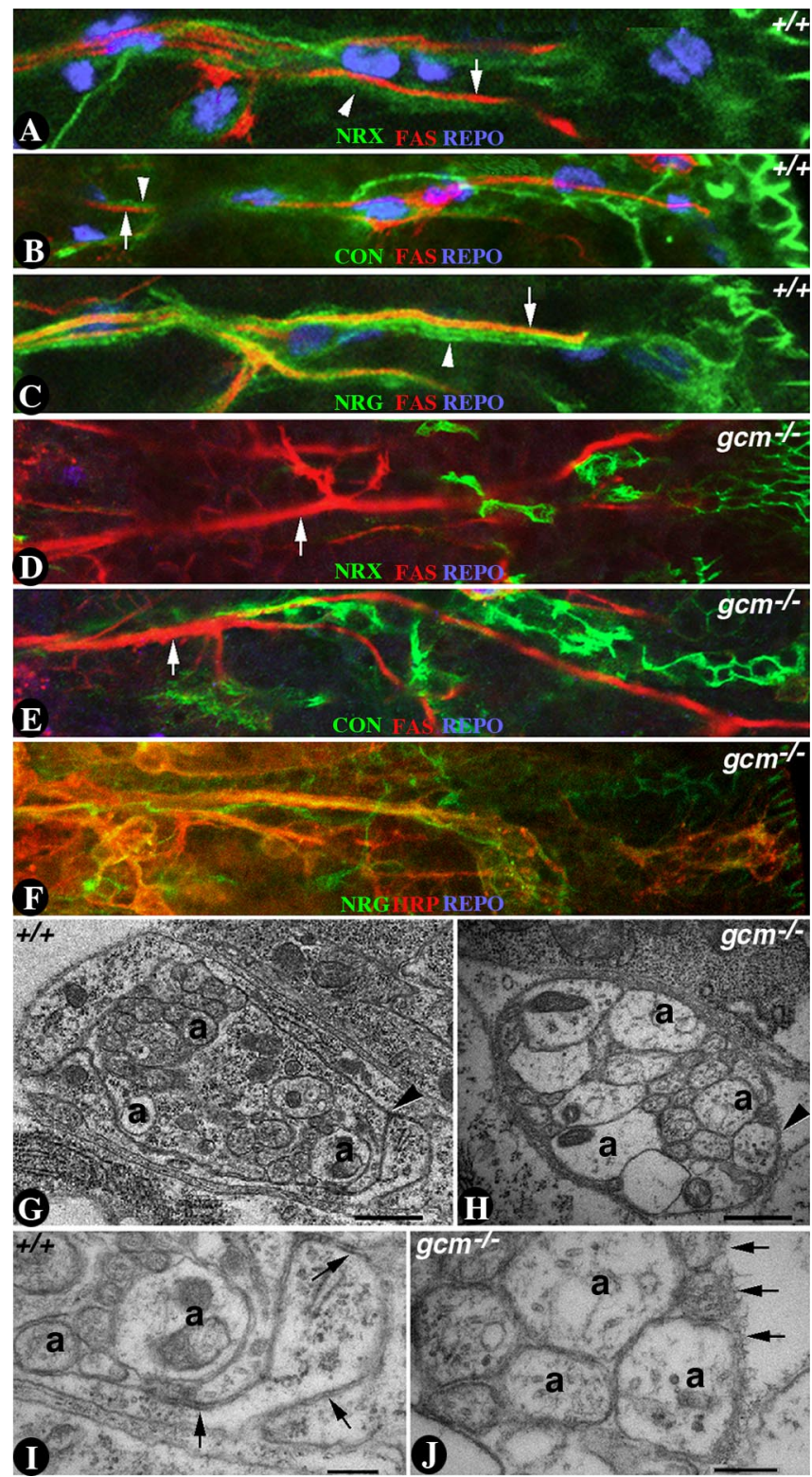

Figure 3. Localization of Nrx IV, Cont, and Nrg in the embryonic nervous system. A, A portion of a wild-type stage 16 embryo stained for Nrx IV (green), Fas II (red), and Repo (blue). Nrx IV localizes to the glial membrane (arrowhead), whereas Fas II stains the motor axons (arrow). The glial nuclei are lined up along the length of the nerve fiber (blue). Cont ( $\boldsymbol{B}$; green, arrowhead) and $\operatorname{Nrg}(\boldsymbol{C} ;$ green, arrowhead) also localize to the glial membrane. However, Nrg has additional localization in the neurons ( $\boldsymbol{C}$; yellow, arrow). $\boldsymbol{D}-\boldsymbol{F}$, A segment of a stage $16 \mathrm{~g}$ cm mutant embryo shows an absence of Repo-positive glial cells (blue) and a lack of glial membrane localization of $\operatorname{Nrx}($ green; $\boldsymbol{D})$, Cont (green; $\boldsymbol{E})$, and Nrg (green, $\boldsymbol{F})$. However, the epithelial localization of Nrx and Cont $(\boldsymbol{D}, \boldsymbol{E}$, respectively) as well as the neuronal localization of $\mathrm{Nrg}(\boldsymbol{F})$ in $\mathrm{gcm}$ mutant embryos remain unaltered (compare with wild type in $\boldsymbol{A}-\boldsymbol{C}$ ). Sensory and motor neurons are stained by anti-HRP (red; $\boldsymbol{F}$ ). G, A cross section through a wild-type embryonic peripheral nerve fiber shows ensheathment of the axons by the glial membrane and close apposition of glial membranes where SJs are observed (black arrowhead). $\boldsymbol{H}$, A cross section through a gcm mutant embryonic peripheral nerve shows the absence of a glial membrane (black arrowhead). However, the axons (a) are still held together as reported previously by Jones et al. (1995). I, A portion of the wild-type nerve from $\mathbf{G}$ at a higher magnification shows glial membranes (black arrows) in close apposition surrounding the axons (a). J, A portion of a gcm mutant peripheral nerve lacks glial membranes (black arrowheads) around the axons (a). Scale bars: $\mathbf{G}, \boldsymbol{H}, 0.5 \mu \mathrm{m} ; \boldsymbol{I}, \boldsymbol{J}, 0.2 \mu \mathrm{m}$.

and outer glial membranes are in close apposition (black arrowhead). In $\mathrm{gcm}$ mutant embryos, no glial membrane is seen surrounding the nerve bundles (Fig. $3 H$ ), and the outer and inner glial membranes are absent (black arrowhead). At a higher mag- 
nification, the wild-type glial membranes (Fig. 3I) closely wrap around the axons (black arrows). The higher magnification of $\mathrm{gcm}$ mutant nerves do not show any glial membranes (Fig. 3J, black arrows), indicating that the membranes around the axons come from the peripheral glial cells. Together, the immunofluorescence and ultrastructural analysis of the wild-type and $\mathrm{gcm}$ mutant embryos further establish that peripheral glial cell membranes are responsible for the ensheathment of the Drosophila peripheral nerves.

\section{Nrx IV, Cont, and Nrg are}

interdependent for their localization in the peripheral nerves

We next studied the localization of Nrx IV, Cont, and Nrg in the embryonic nervous system (Fig. 4A-D). Nrx IV (Fig. 4A), Nrg (Fig. $4 B$ ), and Cont (Fig. 4C) show colocalization at the nerve glial membranes (Fig. $4 D$, white arrows). We have shown previously that Nrx IV, Cont, and Nrg are interdependent for their epithelial SJ localization (Faivre-Sarrailh et al., 2004). Having established that Nrx IV, Cont, and Nrg colocalize in the peripheral nerves, we investigated whether these proteins are interdependent for their localization in the peripheral nerves. We studied the effect of the absence of each of these three proteins on the localization of the other two in the embryonic peripheral nerves using $n r x I V$, cont, and $n r g$ null mutants. $n r x I V$ mutant embryos (Fig. $4 E-H$ ) were stained with anti-Nrx IV (Fig. 4E), anti-Cont (Fig. $4 F$ ), and anti-Nrg (Fig. 4G). Cont and Nrg proteins showed a rather diffused localization when compared with their wild-type localization in Figure 4, $C$ and $B$, respectively. In addition, Cont is present as puncta in the cytoplasm of the glial cells as reported previously for epithelial cells (FaivreSarrailh et al., 2004) because of its failure to get targeted properly to the membrane in the absence of Nrx IV (Fig. $4 H$, arrowheads and inset). Similarly, cont null mutant embryos (Fig. $4 I-L$ ) also showed less defined distribution and reduction of Nrx IV (Fig. $4 J$ ) and Nrg (Fig. $4 K$ ) in the glial membrane (Fig. $4 L$, inset and arrowheads). $n r g$ null mutant embryos (Fig. 4M-P) display significant reduction in Nrx IV (Fig. 4N) and Cont (Fig. 4O) localization in the glial membranes (Fig. $4 P$, arrowheads and inset). These results demonstrate that Nrx IV, Cont, and Nrg are interdependent for their proper localization in the embryonic peripheral nerves.

\section{Nrx IV, Cont, and Nrg exist as a complex in the Drosophila nervous system}

We performed previously ultrastructural analyses of the embryonic epithelia in $n r x I V$, cont, and nrg mutants, which showed that these genes are required for the formation and/or organization of epithelial SJs (Baumgartner et al., 1996; Faivre-Sarrailh et al., 2004). Nrx IV, Cont, and Nrg are interdependent for their proper
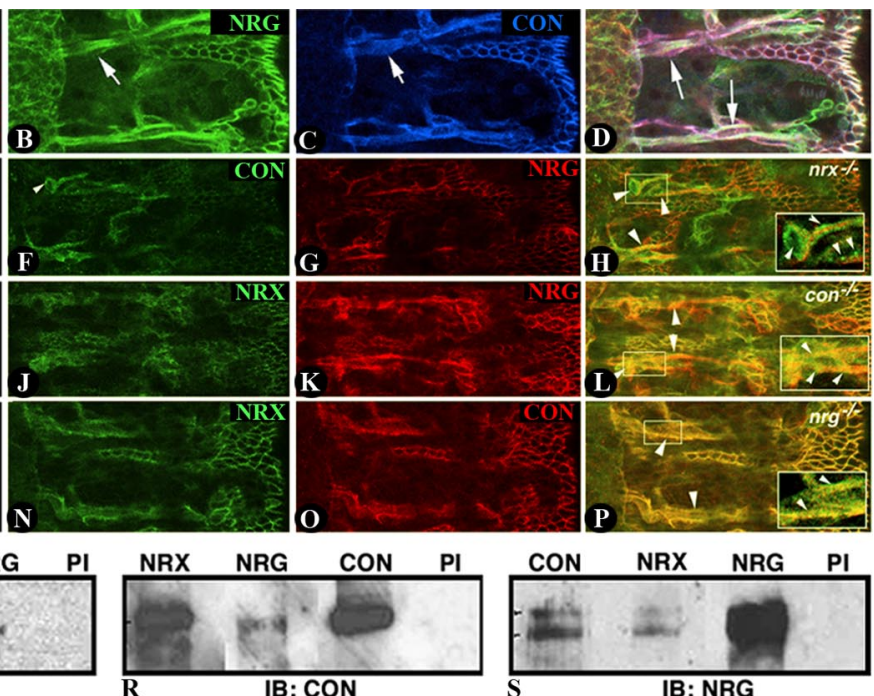

NRX NRG PI
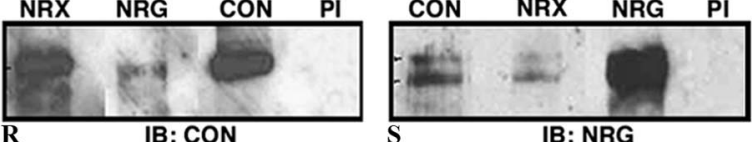

IB: NRG

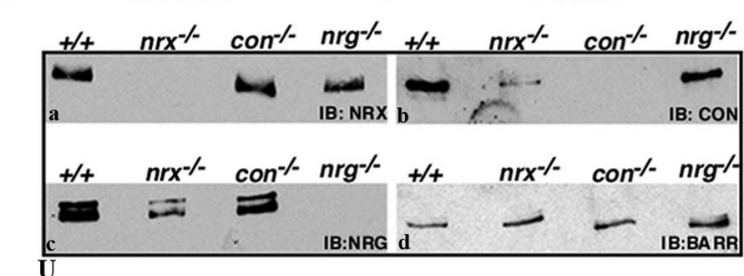

IB: NRG

Figure 4. Interdependence and protein complex formation between $\mathrm{Nrx}$, Cont, and Nrg in the nervous system. A portion of a I iffused localization of Cont $(\boldsymbol{F})$ in the glial membranes (compare with $\boldsymbol{C}$ ). Cont is also localized extensively in small puncta in the (K) (arowheads; instained with anti-Cont (I), anti-Nrx IV $(\boldsymbol{J})$, and anti-Nrg $(\boldsymbol{K})$ shows diffused localization of Nrx IV $(\boldsymbol{J})$ and Nrg (arrowheads; inset in $\boldsymbol{L}$ ), compared with their respective wild-type localization in $\boldsymbol{A}$ and $\boldsymbol{B}$, respectively. $\boldsymbol{M}-\boldsymbol{P}$, A stage $16 \mathrm{nr}$ wild-type $\boldsymbol{A})$ and Cont $(\boldsymbol{O}$ compare with $\boldsymbol{C}$ in the glial membrane (arrowheads; inset in $\boldsymbol{P})$. Coimmunoprecipitation experiments reveal a tripartite complex between Nrx IV, Cont, and Nrg. Adult head lysates were immunoprecipitated with anti-Nrx IV, antiCont, and anti-Nrg antibodies, and the immunoprecipitated proteins were resolved by SDS-PAGE and immunoblotted with anti-Nrx IV (Q), anti-Cont $(\boldsymbol{R})$, and anti-Nrg antibodies (S). All three proteins were immunoprecipitated by their cognate antibod-

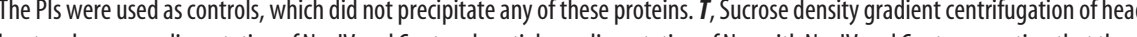
y sates shows cosedimentation of Nrx IV and Cont and partial cosedimentation of Nrg with Nrx IV and Cont, suggesting that these

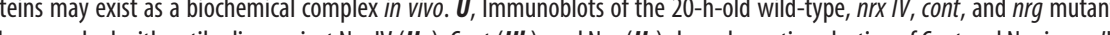
embryos probed with antibodies against $\operatorname{Nrx} I V(\boldsymbol{U a})$, Cont $(\boldsymbol{U b})$, and $\operatorname{Nrg}(\boldsymbol{U c})$ show dramatic reduction of Cont and Nrg in $n r x$ IV mutants. For protein level control, the blots were probed with anti-Barren, an unrelated protein (Ud) (Bhat et al., 1996).

localization both in the epithelia (Faivre-Sarrailh et al., 2004) and in the peripheral nerves, as shown in the preceding section (Fig. 4). These phenotypic similarities raised an interesting possibility that these proteins are part of a macromolecular protein complex that exists in the nervous system. To determine whether these three proteins form a biochemical complex in the nervous system, we used Drosophila heads, which are a rich source of both neurons and glial cells. Coimmunoprecipitation experiments using Nrx IV, Cont, and Nrg antibodies efficiently coprecipitated Nrx IV (Fig. 4Q), Cont (Fig. 4R), and Nrg (Fig. 4S). The preimmune sera (PI) for all of these antibodies were used as controls and did not immunoprecipitate any of these proteins. Interestingly, both isoforms of $\mathrm{Nrg}(180 \mathrm{kDa}$ neuronal and $167 \mathrm{kDa}$ epithelial) were immunoprecipitated by Nrx IV and Cont antibodies, suggesting that both isoforms are part of a protein complex that includes Nrx IV and Cont. However, at this point, we cannot differentiate whether isoform-specific complexes are formed or whether both isoforms are in the same complex. In 

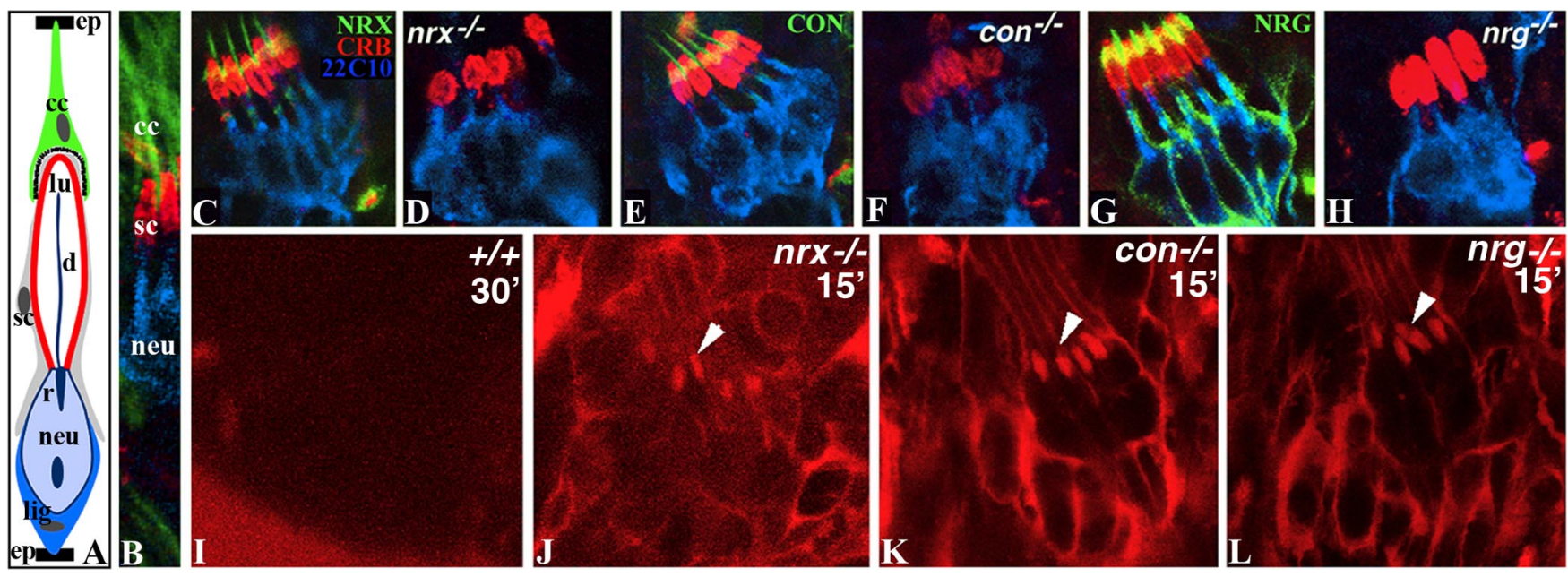

Figure 5. Disruption in CO morphology and BNB function in $n r x I V$, cont, and $n r g$ mutants. $A$, Schematic of a CO showing the various cell types. Cap cells (cc; green), scolopale (s.c.; red), and ligament (lig) are the three glial cell types. The neuron (neu; blue) has a rootlet ( $r$ ), and its dendrite (d) projects into the lumen (lu) of the scolopale. The cc and the lig cell attachment sites of the CO to the epidermis (ep) are also shown. The presence of extensive SJs has been reported between the cap and scolopale cells, thus providing a functional BNB. $\boldsymbol{B}$, Wild-type C0 triple stained with anti- $\beta 3$ tubulin (green) marking the cap cells, anti-Crb (red) marking the lumen of the scolopale, and anti-22C10 (blue) marking the sensory neuron. $\boldsymbol{C}-\boldsymbol{H}$, Wild-type COs (C, $\boldsymbol{E}, \boldsymbol{G})$ stained with anti-CRB (red) and anti-22C10 (blue) in combination with anti-Nrx IV $(\boldsymbol{C}, \boldsymbol{D})$, anti-Cont $(\boldsymbol{E}, \boldsymbol{F})$, and anti-Nrg $(\boldsymbol{G}, \boldsymbol{H})$ show a fusiform shape of the scolopales in the $C 0$ cluster. $n r x$ IV $(\boldsymbol{D})$, cont $(\boldsymbol{F})$, and $n r g(\boldsymbol{H})$ mutants as evident from the lack of staining of their respective antibodies show a defective morphology and a disarrayed organization of the cluster. $I-L$, Dye exclusion assays performed on the wild-type embryos $(\boldsymbol{I}), n r x I V(\boldsymbol{J})$, cont $(\boldsymbol{K})$, and $n r g(\boldsymbol{L})$ mutant embryos. Confocal images after dye injection of the regions of the peripheral nervous system at the level of the $C 0$ s are shown. Wild-type embryos $(\boldsymbol{I})$ excluded the dye from the COs even after $30 \mathrm{~min}$ of injection, indicating that a functional BNB is present. Under identical conditions $n r x I V(\boldsymbol{J})$, cont $(\boldsymbol{K})$, and $n r g(\boldsymbol{L})$ mutant embryos failed to exclude the dye from the COs. Confocal images showed dye penetration into COs within 15 min after injection, indicating that the BNB has broken down in these mutants.

addition, we also performed sucrose density gradient analysis of the fly head lysates to determine how Nrx IV, Cont, and Nrg distribute in buoyant density gradients. As shown in Figure 4T, Nrx IV and Cont cosediment in overlapping fractions (lanes 5-7). Nrg shows distribution in the lighter sucrose density fractions $(3,4)$ that do not overlap with Nrx IV and Cont but partially overlaps with that of Nrx IV and Cont (fractions 5-7), indicating that these proteins are associated with subcellular structures of the same density and may associate into a biochemical complex that is partially maintained during subcellular fractionation.

The immunofluorescence analysis of $n r x I V$, cont, and $n r g$ null mutants showed that in each of these mutants, the other two proteins show qualitatively reduced fluorescence intensities under identical confocal settings (Fig. $4 E-P$ ). The possible explanation for reduced fluorescence intensity could be that loss of any of these proteins affects the localization or stability of the other proteins. We determined whether the levels of the other two proteins had changed in $n r x I V$, cont, and $n r g$ null mutants using immunoblot analysis. As shown in Figure $4 U a$, Nrx IV protein levels did not seem to be affected in cont and $n r g$ mutants when compared with wild type $(+/+)$. Cont protein levels were severely affected in $n r x I V$ mutants compared with wild-type and nrg mutant embryos (Fig. 4Ub). Nrg protein levels were also affected in nrx IV mutants but showed no change in cont mutants (Fig. 4Uc). These changes were not reflected when the same immunoblots were probed with an unrelated protein, Barren (Fig. $4 U d$ ), used as a loading control (Bhat et al., 1996). At this stage, we cannot rule out whether the change in Cont and $\mathrm{Nrg}$ protein levels in $n r x I V$ mutants are attributable to reduced stability or less synthesis of these proteins. Together, the biochemical data indicate that Nrx IV, Cont, and Nrg form a protein complex in the nervous system and that, in the absence of Nrx IV, the stability of Cont and Nrg is severely affected.
Chordotonal organ SJs and blood-nerve barrier defects in $n r x$ $I V$, cont, and $n r g$ mutants

One of the key functions that has been attributed to the SJs in the nervous system is the formation and/or maintenance of a BNB in insects (Baumgartner et al., 1996; Carlson et al., 2000). To test whether Nrx IV, Cont, and Nrg play a role in mediating neuronglial interactions in the embryonic nervous system, we chose lateral chordotonal sensory organs (COs) of the Drosophila PNS because of the presence of both neuronal and glial cell types. We also wanted to test whether disruption in the fine structure of the COs would lead to a breakdown of a functional BNB in the embryonic nervous system. The COs are composed of four cells: cap, scolopale, ligament, and a bipolar neuron. The cap, scolopale, and ligament cells are considered glial cells (Carlson et al., 1997a,b). The anatomical features of a CO highlighting the position and nature of the cells have been schematized in Figure $5 \mathrm{~A}$ based on immunostaining with cell-specific markers. A wild-type $\mathrm{CO}$ is shown highlighting the cap cells by staining with anti- $\beta 3$ tubulin (Fig. 5B, green) (Dettman et al., 2001), scolopale cell lumen, which is labeled with anti-Crb (Fig. 5B, red) (Bhat et al., 1999), and the sensory neuron marked with anti-22C10 (Fig. 5B, blue) (Fujita et al., 1982). The scolopale cell-neuron association forms the core of this organ and provides a functional BNB (Carlson et al., 1997a,b, 2000). As shown in Figure 5C-H, COs were visualized by immunostaining using anti-Crumbs (red) labeling the scolopale and anti-22C10 (blue) labeling the sensory neurons in combination with $\mathrm{Nrx} I V$ (Fig. 5C,D), Cont (Fig. 5E, F), or Nrg (Fig. 5G,H). The Nrx IV, Cont, and Nrg staining revealed that they localize in the scolopale and cap cells, which have been shown to contain SJs and maintain a functional BNB (Carlson et al., 1997a). Nrg is also expressed in the neurons as seen along with 22C10 staining (Fig. 5G). The COs in null mutants of nrx IV (Fig. $5 D$ ), cont (Fig. 5F), and $n r g$ (Fig. 5H) consistently displayed a disorganized morphology and rounded shape of scolopales com- 
pared with the fusiform shape of their respective wild-type counterparts (Fig. 5C, E, and $G$, respectively). The cap cells are present in $n r x I V$, cont, and $n r g$ mutant COs (data not shown). These results suggest that Nrx IV, Cont, and Nrg are not required for the cell specification of the COs but rather for proper neuron-glial interactions during $\mathrm{CO}$ morphogenesis.

To establish whether the anatomical defects observed in the COs of $n r x I V$, cont, and $n r g$ mutants lead to a breakdown of a BNB in the PNS, we performed a dye exclusion assay according to Lamb et al. (1998). Live embryos from late stage 16 or early stage 17 from wild-type, $n r x I V$, cont, and $n r g$ mutants were injected with a $10 \mathrm{kDa}$ rhodamine-labeled dextran dye into the body cavity and monitored for varying periods of time under a confocal microscope. As shown in Figure 5I, wild-type embryos excluded the dye from entering the COs or the nervous system even after 30-60 min of injection. In contrast, mutants for $n r x I V$ (Fig. 5J), cont (Fig. $5 K$ ), and $n r g$ (Fig. $5 L$ ) failed to exclude the dye from COs, and within $15 \mathrm{~min}$ of injection, COs were filled with the dye revealing their anatomical features resulting from dye fluorescence under the confocal microscope. Together, the immunofluorescence and dye exclusion data strongly suggest that Nrx IV, Cont, and Nrg are required for proper $\mathrm{CO}$ morphogenesis and that loss of any one of these proteins results in the breakdown of the BNB in the embryonic nervous system.

$n r x I V$, cont, and $n r g$ null mutants lack glial septate junctions Having established that mutations in $n r x I V$, cont, and $n r g$ show a loss of BNB function in the PNS, we investigated the precise phenotypic defects in the mutant nerves. We performed ultrastructural analysis of $n r x I V$, cont, and $n r g$ null mutants at the level of embryonic peripheral nerve fibers. Ultrastructural analysis of a cross section through a wild-type embryonic nerve reveals close apposition of the outer and inner glial membranes and presence of extensive pleated SJs between these membranes (Fig. 6A, arrows). The inner glial membranes ensheath the axons (Fig. 6, a), which show a clear axolemmal membrane (Fig. 6, arrowheads). An ultrastructure of a comparable cross section through an embryonic nerve of $n r x I V$ null mutants revealed complete loss of SJs between the glial (g) membranes (Fig. 6B, arrows). The inner glial membrane and the axolemma show a fuzzy appearance (black arrowheads), indicating that loss of Nrx IV might also affect the inner glial membrane interactions with the axonal axolemma. The intermembrane spacing between the outer and inner glial membranes did not reveal any change in $n r x I V$ mutant peripheral nerves. These data demonstrate that Nrx IV is critical for establishing glial-glial SJs. cont mutant peripheral nerves also showed a similar loss of glial SJs with no significant change in the intermembrane spacing (Fig. 6G, arrows) as $n r x I V$ mutants (Fig. $1 B$, arrows). Similarly, we performed the ultrastructural analysis of $n$ rg null mutant embryonic peripheral nerves, which revealed an interesting phenotype (Fig. 6D). $n r g$ mutants showed an absence of glial SJs (Fig. 6D,F, arrows) compared with wild-type nerves (Fig. 6C,E, arrows). In addition, the outer and inner glial membranes were more spaced apart compared with the wild-type membranes under identical magnification (Fig. 6, compare $F$ and $E)$. At a higher resolution, the $n r g$ mutant nerves showed parts of the nerve with missing axons and the interaxonal spaces filled with the inner glial cytoplasm (Fig. $6 F$, asterisks). A statistical analysis of the intermembrane spacing between the outer and inner glial membranes of wild-type, $n r x I V$, cont, and $n r g$ mutants $(n=7)$ show that $n r g$ mutants have an average of six times more spacing compared with wild-type nerves (Fig. $6 \mathrm{H}$ ). The outer and inner glial membrane spacing of $n r x$ IV and cont mutant nerves
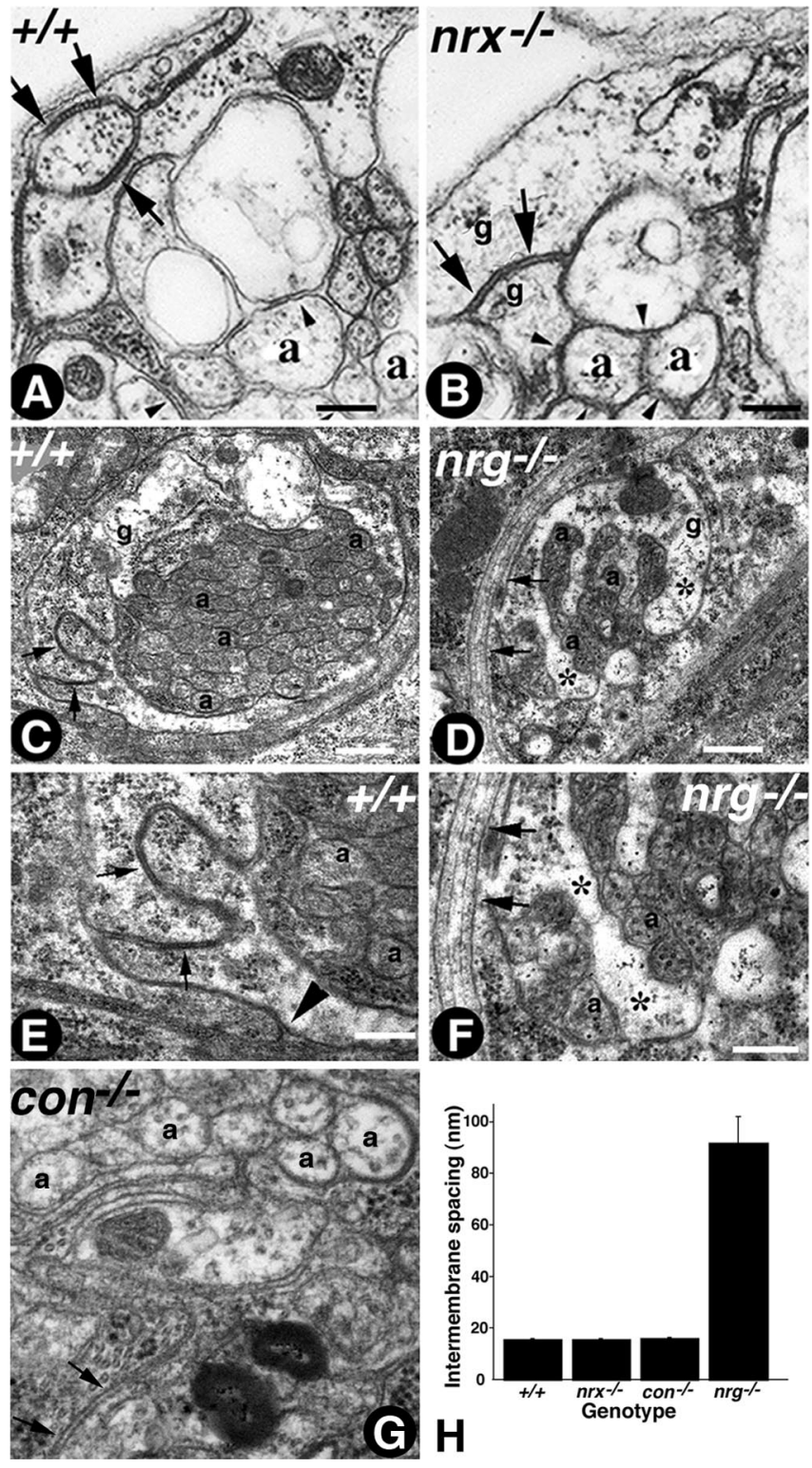

Figure 6. $n r x \mathrm{IV}$, cont, and $n r g$ null mutant embryos lack glial SJs. $\boldsymbol{A}$, Ultrastructure of a portion of a wild-type embryonic nerve shows the presence of distinct ladder-like SJs between the glial membranes (arrows). Also note the smooth membrane surfaces of the axons and the inner glial membrane (arrowheads). B, Ultrastructure of a portion of a nrx IV null mutant embryo shows loss of SJs between the glial membranes (arrows) and a fuzzy appearance at the axon-glial interface (arrowheads). Axons are marked as a, and glial cells are marked as g. C, E, Electron micrograph of a cross section through a wild-type peripheral nerve fiber at increasing magnifications reveals the presence of extensive glial SJs (arrows) and axons (a). D, F, Electron micrograph through a cross section of $n$ rg mutant peripheral nerve fiber at increasing magnifications show complete lack of glial SJs (arrows) and increased spacing between the glial membranes. Note the missing axons in the nrg mutant nerves (asterisks; $\boldsymbol{D}, \boldsymbol{F}$ ). G, Ultrastructure of a cross section of cont mutant peripheral nerve shows loss of glial SJs (arrows) without any change in the intermembrane spacing, a phenotype that is similar to that of $n r x$ IV mutants. $\boldsymbol{H}$, Statistical analysis of glial intermembrane spacing in wild-type, $n r x / V$, cont, and $n r g$ mutant nerves. The intermembrane spacing did not seem significantly different between the wild-type, $n r x I V$, and cont mutants. The intermembrane spacing between the outer and inner glial membranes in nrg mutant showed a dramatic increase when compared with wild-type nerves. Scale bars: $\boldsymbol{A}, \boldsymbol{B}$, E-G, $0.2 \mu \mathrm{m} ; \boldsymbol{C}, \boldsymbol{D}, 0.5 \mu \mathrm{m}$.

were similar to wild type. The phenotypes in $n r g$ mutants suggest that Nrg may help in maintaining the intermembrane distance between the glial membranes by homophilic adhesion and that Nrx IV and Cont along with Nrg then establish SJs. Together, the 
ultrastructural analyses of $n r x I V$, cont, and $n r g$ mutant nerves indicate that Nrx IV, Cont, and Nrg are required to establish SJs between the glial-glial membranes in the Drosophila nervous system.

\section{Discussion}

The ensheathment of nerve fibers provided an evolutionary advantage for species to achieve higher nerve conduction velocities to prey on other species or evade predators to maximize survival. In the present study, we addressed some of the cellular and molecular aspects of axonal insulation in Drosophila PNS. We have shown that inner glial membrane processes are involved in the ensheathment of either an individual axon or a group of axons. This glial ensheathment not only provides insulation of axon/s but also generates unique junctions between either glial membranes or between axons and glial membranes. We showed that Nrx IV, Cont, and Nrg are involved in the establishment of glialglial SJs. Although additional components involved in these interactions need to be identified, our findings as reported here provide a basis for additional analysis of neuronal SJs, which would be relevant to the understanding of their vertebrate counterparts: the paranodal axo-glial SJs (Bhat et al., 2001; Boyle et al., 2001; Bhat, 2003; Hortsch and Margolis, 2003; Salzer, 2003).

\section{Axonal ensheathment: unmyelinated versus myelinated nerve fibers}

The fundamental basis of axonal ensheathment in any species is to faithfully transmit neuronal signals along the nerve fibers and optimize desired cellular responses. To maximize the speed of conduction and/or to minimize the loss of nerve signals, many species evolved mechanisms in which axonal lengths remained short (as seen in insects) by increasing the diameter of the axons or by clustering voltage-gated $\mathrm{Na}^{+}$channels to discrete unmyelinated regions of the axon, the node of Ranvier, as seen in myelinated nerve fibers of vertebrates. Most invertebrate species use some type of glial cells to ensheath their axons without generating a myelin sheath. The insulation is contiguous without any breaks, suggesting that primitive nodal structures or clustering of voltage-gated $\mathrm{Na}^{+}$channels may not exist in invertebrates. However, recent reports have challenged some of these notions. In copepod crustaceans, ultrastructural analysis of the first antenna and the CNS has revealed extensive myelination of sensory and motor axons (Lenz et al., 2000; Weatherby et al., 2000). These studies have raised some fundamental questions about axonal insulation and origins of myelination.

In invertebrates like Drosophila, two types of glial cells are involved in insulation. The inner peripheral glial cells are involved in axonal ensheathment, and the outer (perineurial) glial cells wrap around the inner glial cells to provide another level of ensheathment. This two-cell ensheathment in Drosophila peripheral nerves may be advantageous to ensure that high $\mathrm{K}^{+}$containing hemolymph does not interfere with action potential propagation (Hoyle, 1952). Although the cellular aspects of axonal insulation are being unraveled, the molecular mechanisms underlying the axonal ensheathment remain to be investigated. Most importantly, what are the protein constituents of the insulating membranes, and whether some of the vertebrate myelin proteins have their homologs in invertebrates? A detailed molecular analysis of the nature of the glial cells that ensheath axons as in Drosophila (supplemental Fig. 2S, available at www.jneurosci. org as supplemental material) or produce myelin-like structures and the type of myelin in copepods may provide insights into whether myelinating glial cells arose from a common ancestor.
However, a genetic dissection of the axonal ensheathment in Drosophila will uncover some of the basic aspects of the neuron-glial interactions that lead to ensheathment of nerve fibers across species.

\section{Adhesive interactions between neurons and glia: SJs in flies and vertebrates}

Cell adhesion molecules play a pivotal role in establishing intercellular junctions [e.g., cadherins and associated catenins form a protein scaffold that establish adhesion contacts at the adherens junctions and link them to the actin cytoskeleton (Shan et al., 2004)]. Similarly, claudins and associated cellular scaffolding proteins are required for establishing TJs (Gow et al., 1999; Anderson et al., 2004). In both of these examples, transmembrane proteins bring two opposing membranes together to establish junctions through homophilic and/or heterophilic interactions.

Our finding that $n r g$ null mutant nerves display increased spacing between the outer and inner glial membranes (Fig. 6) suggest that Nrg may be involved in cell-cell interactions and cell-cell adhesion between glial membranes. In contrast, the observation that loss of nrx IV and cont does not affect the membrane spacing between the inner and outer glial membranes suggests that Nrx IV and Cont are not involved in membrane adhesion or bringing the glial membranes in close apposition. Together, these data suggest that Nrg is critical for both the adhesion and SJ formation, whereas Nrx IV and Cont are critical for the formation of the septa at SJs. In addition, the missing axonal fascicles in $n r g$ mutant nerves could result from axon fasciculation defects or axonal degeneration as a secondary consequence resulting from the loss of glial support. The axonal fasciculation defects have been observed previously in $n r g$ mutants (Hall and Bieber, 1997). Alternatively, axonal loss in $n r g$ mutants might result from a disruption in axonal cytoskeleton, because Nrg possesses domains that could potentially interact with and stabilize the axonal cytoskeleton (Cleveland, 1996). Based on Nrg expression in S2 cells, Nrg is predicted to recruit membrane skeleton assembly within specialized domains of neurons in response to cell adhesion (Dubreuil et al., 1996). Both Nrg protein forms $\mathrm{Nrg}^{167}$ and $\mathrm{Nrg}^{180}$ contain a short cytoplasmic domain as a binding site for ankyrin (Hortsch et al., 1998). Ankyrins are linker proteins that connect various membrane proteins with the actinspectrin network in the cell (Bouley et al., 2000). Loss of axons observed in $n r g$ mutants is clearly suggestive of axon-glial interdependence that may alter axonal survival. In vertebrates, axolemmal-myelin interactions are critical for the formation of the paranodal axo-glial SJs (Bhat, 2003; Banerjee et al., 2006; Garcia-Fresco et al., 2006). This raises an interesting possibility that axon-glial interactions in Drosophila may use similar interactive mechanism for establishing axo-glial SJs (supplemental Fig. S2, available at www.jneurosci.org as supplemental material). Thus, our studies on Nrx IV, Cont, and Nrg suggest that these proteins are critical for the formation and/or organization of the SJs between either glial cells and possibly between axons and glial cells, which remains to be further investigated.

\section{SJs as molecular barriers: a common theme in axonal insulation}

Invertebrate axons are insulated from their salt-rich environment through a glial-dependent BBB, which plays a crucial role in electrical and chemical insulation (Auld et al., 1995; Baumgartner et al., 1996; Bainton et al., 2005; Schwabe et al., 2005). Ultrastructural studies have demonstrated SJs between perineurial glial cells and inner glial cell membrane to form the structural basis of BBB 
of some insects (Carlson et al., 2000). As reported in this study, absence of SJs in $n r x I V$, cont, and $n r g$ mutants (Fig. 6), and a compromised BNB in the PNS as evidenced by dye exclusion analyses (Fig. 5), provide additional confirmation in support of SJs as a prerequisite for BNB formation. Not just in the PNS, recent reports on the $\mathrm{BBB}$ formation and function also in the CNS of Drosophila underscore the importance of SJs in proper sealing and insulation of the nerve cord (Schwabe et al., 2005), thereby supporting that insulation and establishment of functional SJs in both PNS and CNS go hand in hand. Although, G-protein-coupled receptor signaling pathway members have been identified to establish the BBB in Drosophila CNS, the signaling mechanisms that operate in the PNS still remain to be established.

\section{Drosophila neural SJs and vertebrate axo-glial SJs: molecular similarities}

The axo-glial SJs in the myelinated axons share many anatomical features similar to those of invertebrate SJs, especially the electron-dense ladder-like transverse septa (Bhat, 2003; Banerjee et al., 2006). Ensheathment of Drosophila axons by perineurial glial cells in the absence of myelin-producing glial cells would have predicted that the molecular components of the invertebrate SJs would be different from those of the vertebrate axo-glial SJs. Surprisingly, the fly SJ molecular components are present at the vertebrate axo-glial SJs and not at the TJs, which serve similar functions. Thus, the molecular similarities reflect an evolutionarily conserved function of creating an ionic barrier both at Drosophila SJs and at axo-glial SJs in the paranodal region (Rios et al., 2003). More importantly, Drosophila nerves contain a large number of axons, which are collectively held together by glial membranes not only to maintain nerve fasciculation but also to maintain the insulation of individual axons. It would be of significant interest to establish whether axo-glial SJs are present in Drosophila nerves (Jia et al., 1993; Juang and Carlson, 1994; Banerjee et al., 2006) (supplemental Fig.S2, available at www.jneurosci.org as supplemental material) and to identify downstream components of these junctions in Drosophila that link these junctions to the glial or axonal cytoskeleton. Identification of such molecules will provide insights into whether these junctions play a much broader role in axon-glial signal transduction. In summary, the molecular and functional similarities between the Drosophila SJs and vertebrate axo-glial SJs should allow us to undertake a genetic and molecular dissection of the formation and function of these junctions in Drosophila.

\section{References}

Anderson JM, Van Itallie CM, Fanning AS (2004) Setting up a selective barrier at the apical junction complex. Curr Opin Cell Biol 16:140-145.

Auld VJ, Fetter RD, Broadie K, Goodman CS (1995) Gliotactin, a novel transmembrane protein on peripheral glia, is required to form the bloodnerve barrier in Drosophila. Cell 81:757-767.

Bainton RJ, Tsai LT, Schwabe T, DeSalvo M, Gaul U, Heberlein U (2005) moody encodes two GPCRs that regulate cocaine behaviors and bloodbrain barrier permeability in Drosophila. Cell 123:145-156.

Banerjee S, Sousa AD, Bhat MA (2006) Organization and function of septate junctions: an evolutionary perspective. Cell Biochem Biophys, in press.

Baumgartner S, Littleton JT, Broadie K, Bhat MA, Harbecke R, Lengyel JA, Chiquet-Ehrismann R, Prokop A, Bellen HJ (1996) A Drosophila neurexin is required for septate junction and blood-nerve barrier formation and function. Cell 87:1059-1068.

Bellen HJ, Lu Y, Beckstead R, Bhat MA (1998) Neurexin IV, caspr and paranodin-novel members of the neurexin family: encounters of axons and glia. Trends Neurosci 21:444-449.

Bhat MA (2003) Molecular organization of axo-glial junctions. Curr Opin Neurobiol 13:552-559.
Bhat MA, Philp AV, Glover DM, Bellen HJ (1996) Chromatid segregation at anaphase requires the barren product, a novel chromosome-associated protein that interacts with Topoisomerase II. Cell 87:1103-1114.

Bhat MA, Izaddoost S, Lu Y, Cho KO, Choi KW, Bellen HJ (1999) Discs Lost, a novel multi-PDZ domain protein, establishes and maintains epithelial polarity. Cell 96:833-845.

Bhat MA, Rios JC, Lu Y, Garcia-Fresco GP, Ching W, St Martin M, Li J, Einheber S, Chesler M, Rosenbluth J, Salzer JL, Bellen HJ (2001) Axonglia interactions and the domain organization of myelinated axons requires neurexin IV/Caspr/Paranodin. Neuron 30:369-383.

Bouley M, Tian MZ, Paisley K, Shen YC, Malhotra JD, Hortsch M (2000) The L1-type cell adhesion molecule neuroglian influences the stability of neural ankyrin in the Drosophila embryo but not its axonal localization. J Neurosci 20:4515-4523.

Boyle ME, Berglund EO, Murai KK, Weber L, Peles E, Ranscht B (2001) Contactin orchestrates assembly of the septate-like junctions at the paranode in myelinated peripheral nerve. Neuron 30:385-397.

Campbell G, Goring H, Lin T, Spana E, Andersson S, Doe CQ, Tomlinson A (1994) RK2, a glial-specific homeodomain protein required for embryonic nerve cord condensation and viability in Drosophila. Development 120:2957-2966

Campos-Ortega JA, Hartenstein V (1997) The embryonic development of Drosophila melanogaster. Ed 2. New York: Springer.

Carlson SD, Hilgers SL, Juang JL (1997a) First developmental signs of the scolopale (glial) cell and neuron comprising the chordotonal organ in the Drosophila embryo. Glia 19:269-274.

Carlson SD, Hilgers SL, Juang JL (1997b) Ultrastructure and blood-nerve barrier of chordotonal organs in the Drosophila embryo. J Neurocytol 26:377-388.

Carlson SD, Juang JL, Hilgers SL, Garment MB (2000) Blood barriers of the insect. Annu Rev Entomol 45:151-174.

Cleveland DW (1996) Neuronal growth and death: order and disorder in the axoplasm. Cell 84:663-666.

Dettman RW, Turner FR, Hoyle HD, Raff EC (2001) Embryonic expression of the divergent Drosophila beta3-tubulin isoform is required for larval behavior. Genetics 158:253-263.

Dubreuil RR, MacVicar G, Dissanayake S, Liu C, Homer D, Hortsch M (1996) Neuroglian-mediated cell adhesion induces assembly of the membrane skeleton at cell contact sites. J Cell Biol 133:647-655.

Faivre-Sarrailh C, Banerjee S, Li J, Hortsch M, Laval M, Bhat MA (2004) Drosophila contactin, a homolog of vertebrate contactin, is required for septate junction organization and paracellular barrier function. Development 131:4931-4942.

Freeman MR, Delrow J, Kim J, Johnson E, Doe CQ (2003) Unwrapping glial biology: Gcm target genes regulating glial development, diversification, and function. Neuron 38:567-580.

Fujita SC, Zipursky SL, Benzer S, Ferrus A, Shotwell SL (1982) Monoclonal antibodies against the Drosophila nervous system. Proc Natl Acad Sci USA 79:7929-7933.

Garcia-Fresco GP, Sousa AD, Pillai AM, Moy SS, Crawley JN, Tessarrollo L, Dupree JL, Bhat MA (2006) Disruption of axo-glial junctions causes cytoskeletal disorganization and degeneration of Purkinje neuron axons. Proc Natl Acad Sci USA, in press.

Gow A, Southwood CM, Li JS, Pariali M, Riordan GP, Brodie SE, Danias J, Bronstein JM, Kachar B, Lazzarini RA (1999) CNS myelin and sertoli cell tight junction strands are absent in Osp/claudin-11 null mice. Cell 1999 99:649-659.

Hall SG, Bieber AJ (1997) Mutations in the Drosophila neuroglian cell adhesion molecule affect motor neuron pathfinding and peripheral nervous system patterning. J Neurobiol 32:325-340.

Hodes R (1953) Linear relationship between fiber diameter and velocity of conduction in giant axon of squid. J Neurophysiol 16:145-154.

Hortsch M, Margolis B (2003) Septate and paranodal junctions: kissing cousins. Trends Cell Biol 13:557-561.

Hortsch M, Homer D, Malhotra JD, Chang S, Frankel J, Jefford G, Dubreuil RR (1998) Structural requirements for outside-in and inside-out signaling by Drosophila neuroglian, a member of the L1 family of cell adhesion molecules. J Cell Biol 142:251-261.

Hosoya T, Takizawa K, Nitta K, Hotta Y (1995) Glial cells missing: a binary switch between neuronal and glial determination in Drosophila. Cell 82:1025-1036. 
Hoyle G (1952) High blood potassium in insects in relation to nerve conduction. Nature 169:281-282.

Ito K, Urban J, Technau GM (1995) Distribution, classification and development of Drosophila glial cells in the late embryonic and early larval ventral nerve cord. Roux's Arch Dev Biol 204:289-307.

Jia XX, Gorczyca M, Budnik V (1993) Ultrastructure of neuromuscular junctions in Drosophila: comparison of wild type and mutants with increased excitability. J Neurobiol 24:1025-1044.

Jones BW, Fetter RD, Tear G, Goodman CS (1995) Glial cells missing: a genetic switch that controls glial versus neuronal fate. Cell 82:1013-1023.

Juang JL, Carlson SD (1994) Analog of vertebrate anionic sites in bloodbrain interface of larval Drosophila. Cell Tissue Res 277:87-95.

Lamb RS, Ward RE, Schweizer L, Fehon RG (1998) Drosophila coracle, a member of the protein 4.1 superfamily, has essential structural functions in the septate junctions and developmental functions in embryonic and adult epithelial cells. Mol Biol Cell 9:3505-3519.

Leiserson WM, Harkins EW, Keshishian H (2000) Fray, a Drosophila serine/ threonine kinase homologous to mammalian PASK, is required for axonal ensheathment. Neuron 28:793-806.

Lenz PH, Hartline DK, Davis AD (2000) The need for speed. I. Fast reactions and myelinated axons in copepods. J Comp Physiol A Neuroethol Sens Neural Behav Physiol 186:337-345.

Peters A, Palay SL, Webster HD (1991) The fine structure of the nervous system: neurons and their supporting cells, Ed 3. New York: Oxford.
Rios JC, Rubin M, St Martin M, Downey RT, Einheber S, Rosenbluth J, Levinson SR, Bhat M, Salzer JL (2003) Paranodal interactions regulate expression of sodium channel subtypes and provide a diffusion barrier for the node of Ranvier. J Neurosci 23:7001-7011.

Salzer JL (2003) Polarized domains of myelinated axons. Neuron 40:297-318.

Schwabe T, Bainton RJ, Fetter RD, Heberlein U, Gaul U (2005) GPCR signaling is required for blood-brain barrier formation in Drosophila. Cell 123:133-144.

Sepp KJ, Schulte J, Auld VJ (2000) Developmental dynamics of peripheral glia in Drosophila melanogaster. Glia 30:122-133.

Shan W, Yagita Y, Wang Z, Koch A, Svenningsen AF, Gruzglin E, Pedraza L, Colman DR (2004) The minimal essential unit for cadherin-mediated intercellular adhesion comprises extracellular domains 1 and 2. J Biol Chem 279:55914-55923.

Tepass U, Tanentzapf G, Ward R, Fehon R (2001) Epithelial cell polarity and cell junctions in Drosophila. Annu Rev Genet 35:747-784.

Weatherby TM, Davis AD, Hartline DK, Lenz PH (2000) The need for speed. II. Myelin in calanoid copepods. J Comp Physiol A Neuroethol Sens Neural Behav Physiol 186:347-357.

Williams DW, Tyrer M, Shepherd D (2000) Tau and tau reporters disrupt central projections of sensory neurons in Drosophila. J Comp Neurol 428:630-640. 